\title{
Motor and cognitive development: the role of karate
}

\author{
Marianha Alesi ${ }^{1}$ \\ Antonino Bianco ${ }^{2}$ \\ Johnny Padulo3,4 \\ Francesco Paolo Vella ${ }^{5}$ \\ Marco Petrucci \\ Antonio Paoli6 \\ Antonio Palma ${ }^{2}$ \\ Annamaria Pepi ${ }^{1}$ \\ 1 Department of Psychology, University of Palermo, \\ Italy \\ 2 Sport and Exercise Sciences Research Unit, Uni- \\ versity of Palermo, Italy \\ 3 Sport Science, University e-Campus, Novedrate, Italy \\ 4 Tunisian Research Laboratory "Sport Performance \\ Optimization" National Center of Medicine and Sci- \\ ence in Sport, Tunis, Tunisia \\ 5 Italian Federation of Judo, Wrestling, Karate, Mar- \\ tial Arts, FIJIKam-Coni, Italy \\ 6 Department of Biomedical Science, University of \\ Padua, Italy
}

Corresponding author:

Johnny Padulo

Sport Science, University e-Campus

Via Isimbardi, 10

22060 Novedrate, Italy

E-mail: sportcinetic@gmail.com

\section{Summary}

Background: regular physical activity has an effect on biological responses in both muscles and organs that, in turn, alter the structure and functions of the brain. Therefore, this study aims at comparing motor (sprint, coordination ability and explosive legs strength skills) and cognitive abilities (working memory, attention, executive functioning) in children. Methods: 39 children with average chronological age of 9 years were divided in: Karatekas $(n=19)$ and Sedentary $(n=20)$ groups. Their abilities were measured by motor and cognitive tests. Motor skills were assessed through a battery composed by the $20 \mathrm{mt}$ Sprint test, the Agility test and the Standing board jump Test. Cognitive profile was assessed by a battery of tests derived from BVN 5-11, "Batteria di Valutazione Neuropsicologica per l'Età Evolutiva": Visual discrimination test, Reaction time test, Forwards and Backwards Digit Span Tests, Corsi Block-Tapping test and Tower of London. Results: our results reveal significant differences between two groups $(p<0.05)$. Karate children show better speed times, explosive legs strength and coordination skills. They scored better on working memory, visual selective attention and executive functions. Conclusion: karate exercise training shows global benefits resulting in physiological and psychological gains in children.

KEY WORDS: children, cognitive abilities, motor abilities, motor control, field testing.

\section{Introduction}

A growing amount of research pointed out that regular physical activity has an effect on biological responses in both muscles and organs that, in turn, alter the structure and functions of the brain ${ }^{1}$. This assumption stems from a multidisciplinary approach which brings together research from different disciplines such as kinesiology, human anatomy, neurosciences. It is worth noting the increasing interest of developmental and educational psychology to focus on the aspects concerning the contextual effects of the synergy body-mind during the life span. Historically, sport science emphasized the Athletes' physical characteristics in order to maximize their sport performance; consequently the main work of the trainers aimed at developing physical and technical qualities of athletes ${ }^{2}$. It's largely recognized that athletes show better competences in skills such as speed, force, ability to jump higher, accuracy and efficiency of motor responses. Recently these assumptions have been corroborated by neuroscience studies using non-invasive neurophysiological and neuroimaging methods aimed at deepening both motor and cognitive processing ${ }^{3}$. The first ones enclose preparation, execution, and imagery, the last ones embrace neural activities, such as perception, stimulus discrimination, decision making ${ }^{2}$. From the developmental perspective, the study of the adult athlete's brain reveals to be particularly suitable to analyze in depth the brain neuroplasticity given their long-term training and practice, often starting very early in childhood. More specifically studies of developmental neuropsychology demonstrate the close relation between physical fitness and brain development and functioning from early childhood ${ }^{4,5}$. This relationship would be mediated by physiological and psychological mecha- 
nisms; an adequate physical fitness concerning situps, push-ups, long jump, hand grip, the cardiorespiratory efficiency (50-m sprint, $1.6 \mathrm{~km}$ run, and sub-maximal measure of $\mathrm{VO}_{2}$ ), and motor general activities promote children's gains in specific cognitive functioning and goal-directed behaviours ${ }^{1,4}$. To date, the literature supports the causal link between regular physical activity and brain development particularly in the prefrontal cortical area.

More in depth, physical activity would act by enhancing the production of neurotrophins, the synaptogenesis and the angiogenesis with the consequent improvement in cognitive performances such as speed of processing, planning and control strategies employ, working memory ${ }^{5,6}$. This effect is particularly strong on the executive functions, involved in planning and selecting strategies that regulate and control goal directed behaviours such as encoding, stimulus evaluation, response selection or inhibition and response execution. It's hypothesized that the executive functioning can be influenced by physical and sport activities in children too $0^{5,7}$. Consequently it's crucial for researchers and practitioners to identify what sport activities are able to improve the development both in motor and cognitive domain. In this perspective, karate revealed to be a physical activity able to stimulate memory abilities and executive functioning and not simple technical elements during training sessions $^{8}$. Karate "Kumite" is a full contact sport that focuses on the philosophical, moral, and ethical principals characterizing the martial arts. Particularly it includes a combination of techniques used in the competition between two opponents aimed at the disqualification. Given its nature of discipline focusing on selfprotection and health enhancement, karate would be considered as sport and therapy at once ${ }^{9}$.

In their recent review Chaabène et al. ${ }^{10}$ describe the most significant physical and physiological characteristics of karate athletes. They report the importance of an high fitness, muscle power at lower loads, vertical jump performance, maximal power and maximal velocity, flexibility ${ }^{10}$. The authors shed additional light on the reaction time as core element in karate because top-level performance is largely supported by explosive techniques ${ }^{10}$. On the other hand this conjunction between motor capacity and cognitive variables such as reaction time is coherent with the cardiovascular fitness hypothesis ${ }^{11}$ concerning the influence of physical activity on cognitive functioning. In particular, this influence would be mediated by two mechanisms, the decrease of disease related to diabetes, hypertension, and cardiovascular illness and the increase in brain structure and function.
Consistent with this hypothesis, since martial arts enhance aerobic performance capacity, they could also favour the cognitive functioning including reaction time and motor time, information processing speed, working memory ${ }^{1,4}$. Chateau-Degat et al. ${ }^{12}$ assessed improvements in motor skills and cognitive functions following a course of karate training over a one-year in elderly people. They found gains in tactic skills and motor speed. Moreover this training program affects positively the performance on tasks based on reaction time and anticipatory skills with improvement in information processing and visual-motor coordination. The positive effects of regular karate practice were maintained at follow-up assessment after one year ${ }^{12}$. From a developmental perspective the above-mentioned gain in reaction time skills is consistent with similar findings obtained among young regularly practicing karate when compared with novice peers ${ }^{13}$. Consequently it is plausible that the benefits of karate could be extended to predict improvements in children's motor abilities and cognitive functions. In view of the above, this research investigates the key role played by karate in motor and cognitive abilities in childhood. The main aim is to explore the motor abilities of children regularly (3 times a week) practicing karate with reference to children having a sedentary life-style. A further aim of the study is to compare the cognitive profile of children practicing karate with that of sedentary children of the same chronological age. It was expected that children involved in karate would have better motor abilities and cognitive abilities than sedentary children with specific motor abilities concerning run, coordination skills and explosive legs strength. On the other hand, the cognitive profile implicated short-term memory (verbal and visual-spatial memory), attention (visual discrimination and visual selective attention) and executive functions. The final aim of the study was to verify the relationship between motor and cognitive abilities.

\section{Materials and methods}

\section{Subjects}

In this research participated 39 children (Tab. 1) regularly attending karate (mainly kumitè) courses or having sedentary life-style. The sport group consisted of 19 children (55\% males and $45 \%$ females), with an average chronological age of 9.05 years and training experience ranging from 3 to 5 years with three karate training session per week (total $3<4$ hours per week). The sedentary group (40\% males and $60 \%$ fe-

Table 1. Anthropometric data.

\begin{tabular}{lllll}
\hline Subjects & Age (years) & Weight $\mathbf{( k g )}$ & Height $\mathbf{( c m )}$ & BMI $\left(\mathbf{k g} / \mathbf{m}^{2}\right)$ \\
\hline Karate & $9.05 \pm 1.04$ & $31.59 \pm 6.69$ & $1.35 \pm 1.21$ & $17.07 \pm 1.74$ \\
Sedentary & $9.15 \pm 0.99$ & $33.12 \pm 7.02$ & $1.34 \pm 1.15$ & $18.4 \pm 1.82$ \\
\hline
\end{tabular}

Anthropometric data $($ mean $\pm S D)$ in Karate $(n=19)$ and Sedentary $(n=20)$ groups for age, weight, height and BMI. 
males) consisted of the remaining 20 children with an average chronological age of age 9.15 years. Prior to the start of the study, parents' written informed consent for each child was obtained. The study was performed in compliance with the basic principles and recommendations in clinical and field science published. All experimental procedures were approved by the University Human Research Ethics Committee, which followed the ethical standard of the Muscle, Ligament and Tendons Journal ${ }^{14}$.

Experimental set-up

Prior to the administration of motor and cognitive tests, anthropometric measurement was realized in order to assess body weight and height for all children. Anthropometric and motor measurements were done during one session (physical/motor session) and cognitive evaluation during another session. The second session was carried out over 2 weeks and the order of presentation of the two sessions was balanced. Both the physical/motor session and the cognitive session were realized in the gym.

The body mass index (BMI) was calculated as bodyweight divided by height squared $\left(\mathrm{kg} \cdot \mathrm{m}^{2}\right)$. Motor skills were assessed through a battery composed by the $20 \mathrm{mt}$ Sprint test, the Agility test and the Standing board jump Test. Specifically with regard to $20 \mathrm{mt}$ Sprint test, was measured the sprint time by chronometer. After the demonstration each child was asked to run as fast as possible.

The Agility test measured the coordination skills along a circuit with hurdles: from the starting line the child had to overcome a central cone, turn right and reach the first obstacle of $50 \mathrm{~cm}$ in height, overcome with a leap and immediately go under the same in the opposite direction, return to the central cone and repeat the procedure in the four cardinal directions. Children were required to complete this circuit as first as possible and without errors during their performance. The score was expressed in seconds calculated by the chronometer. The start was taken when one of the feet has already left the floor while the end of registration was taken when one part of participants' body crossed the Stop line (Fig. 1).

Finally the Standing board jump test measured the children explosive legs strength. Each child was asked to jump horizontally as far as he could land with both feet from a standing position. The score was expressed in meters. For each motor test were done two trials and was considered the best performance.

Cognitive profile was assessed by a battery of tests derived from BVN 5-11 "Batteria di Valutazione Neuropsicologica per l'Età Evolutiva"15: Visual discrimination test, Reaction time test, Forwards and Backwards Digit Span Tests, the Corsi Block-Tapping test and the Tower of London. The Visual Discrimination Test assessed the ability to identify correctly stimuli by finding likenesses and differences in stimuli. Given a target, children had to recognize all similar figures within a matrix of 9 shapes. The parameters of evaluation were two: the correct items and the time of exe-

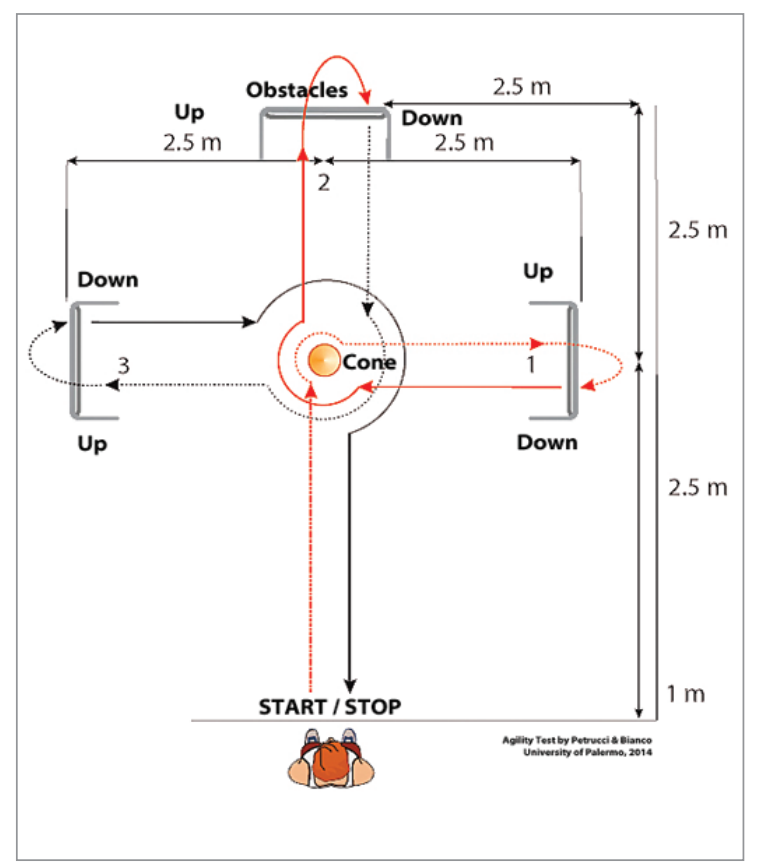

Figure 1. The Figure is representing the agility test. Nearby the start/stop side all participants have $1 \mathrm{~m}$ of margin in order to get prepared. As you can see there is equal distance $(2.5 \mathrm{~m})$ from the central cone of the agility test. The numbers reported without unit of measure are representing the order of execution (Start, 1, 2, 3, Stop).

cution indicated in seconds. For each item the score was 0 in the case of incorrect execution or 1 in the case of correct execution. Therefore, the total score ranged from 0 to 9 points. The Visual Selective Attention test was an object decision task aimed at evaluating the object knowledge and recognition.

It was a paper-and-pencil cancellation task requiring the subject to select and cross out targets as quickly as possible (within 1 minute). The targets were geometrical figures represented by squares with two lines inside and embedded in a frame containing 64 randomly positioned items of which 52 were distractors. The total number of correct choices was computed and the maximum score was 12 points. With concern the memory tests, the Forwards Digit Span Test consisted of a list of 27 sequences of numbers with increasing level of difficulty. The child was required to repeat immediately and in the same order the list of numbers verbally presented by the experimenter. The score was defined by the number of items correctly remembered. The Backwards Digit Span Test consisted of a list of 24 sequences of numbers with increasing level of difficulty according to the length for each trial. The child had to repeat immediately and in the backwards order the list of numbers verbally presented by the experimenter. Procedures of scoring were similar to Forward Digit Span Test. The Corsi Block-Tapping test measured the visual-spatial sketchpad. In consisted in a wooden boards with wooden blocks $(4.5 \mathrm{~cm} \times 4.5 \mathrm{~cm})$ numbered on the side visible only to the experimenter. The child was required to 
observe the sequence of blocks "tapped" by the researcher in a scrambled order, to remember and reproduce immediately the sequences with an increasing level of difficulty. The score was defined by the number of visual-spatial items correctly remembered and ranged from 2 to 7 . The Tower of London test assessed the executive functioning. It consisted of wooden platform with three vertical rods and three colored balls (red, green, and blue). The child had to move the three spheres, starting from an initial position to different final positions. The task was composed by 12 items expressed in target positions with an increasing level of difficulty. The total score ranged from 0 to 12 points.

\section{Statistical Analysis}

The Univariate analyses of variance (ANOVA) and "Fisher" test was performed to investigate the comparisons between the motor and cognitive abilities in children regularly involved in karate and sedentary children. The independent variable was the sport activities/sedentary life style, whilst the dependent ones were motor abilities such as run, coordination and explosive legs strength and cognitive abilities such as attention, memory and executive functions. Pearson's correlation test was performed to assess the association between motor and cognitive skills. The level of significance was set at $p<0.05$ and all data are presented as Mean and SD. The test-retest reliability was obtained through the Intra-class Correlation Coefficient (ICC). The SPSS 20 Software was adopted for this analysis.

\section{Results}

The ICC of the visual discrimination scale was 0.99 , like the visual selective attention value (0.99). The forwards digit span tests showed an ICC of 0.84 while the tower of London was a bit lower (0.75). The backwards digit span test and the Corsi block-tapping test showed ICC values of 0.81 and 0.84 , respectively. The Table 2 summarizes the normative means and standard deviations, separately for karatekas and sedentary children, on each measure from motor tasks. Results revealed significant differences between the two groups for all motor tasks: $20 \mathrm{mt}$ sprint $\left[\mathrm{F}_{(1,38)}=19.492 ; \mathrm{p}<0.001\right]$, agility test $\left[F_{(1,38)}=51.384 ; p<0.001\right]$ and standing board jump $\left[F_{(1,38)}=19.537 ; p<0.001\right]$. Children attending karate demonstrated higher competences in the run, in the coordination and in the explosive legs strength than their peers.

The Table 3 summarizes the normative means and standard deviations, separately for karatekas and sedentary children, on each measure from cognitive tasks. Data analysis showed significant differences between the two groups for the following abilities: $\mathrm{Vi}$ sual Selective Attention $\left[F_{(1,38)}=5.486 ; p<0.05\right]$, Forward Digit Span $\left[F_{(1,38)}=6.133 ; p<0.05\right]$, Backward Digit Span $\left[F_{(1,38)}=14.790 ; p<0.05\right]$, Executive Functions $\left[F_{(1,38)}=4.172 ; p<0.05\right]$ and Times of Executive Functions $\left[F_{(1,37)}=7.248 ; p<0.05\right]$.

On the whole, children attending karate appear to have better cognitive functioning. In other words, they show higher scores in visual selective attention task, in working memory both for forward digit span and backward digit span. Moreover data analysis revealed significant differences between the two groups in all both the measures of Tower of London Test; children attending karate show better execution of planning tasks and lower times of planning tasks.

Concerning the relationship between motor and cognitive abilities, our analysis revealed two significant ( $p$ $<0.05$ ) associations only in the sport group: one negative correlation between the time of performance on Visual Discrimination Test and Standing board jump Test $(r=-0.583)$ and one positive correlation between the time of performance on Visual Discrimination and the time of execution on agility test $(r=0.530)$.

Table 2. Motor skills performance.

\begin{tabular}{llll}
\hline Variable & Karate children & Sedentary children & Comparison (p value) \\
\hline $20 \mathrm{mt}$ Sprint $(\mathrm{Sec})$ & $4.18 \pm 0.23$ & $4.84 \pm 0.57$ & 0.001 \\
Agility (Sec) & $15.78 \pm 1.87$ & $22.93 \pm 3.73$ & 0.001 \\
Standing broad jump (cm) & $135.82 \pm 19.69$ & $101.71 \pm 26.12$ & 0.001
\end{tabular}

Descriptive statistics for $20 \mathrm{mt}$ Sprint, agility and standing broad jump.

Table 3. Cognitive skills performance.

\begin{tabular}{llllll}
\hline Group & $\begin{array}{l}\text { Forward } \\
\text { Digit Span }\end{array}$ & $\begin{array}{l}\text { Backward } \\
\text { Digit Span }\end{array}$ & $\begin{array}{l}\text { Selective } \\
\text { Attention }\end{array}$ & $\begin{array}{l}\text { London } \\
\text { Tower Test }\end{array}$ & $\begin{array}{l}\text { London } \\
\text { Tower Test }\end{array}$ \\
\hline Karate & $5.37 \pm 1.26$ & $3.58 \pm 0.77$ & $9.74 \pm 1.37$ & $9.84 \pm 1.71$ & $11.92 \pm 4.42$ \\
Sedentary & $4.55 \pm 0.76$ & $2.70 \pm 0.66$ & $7.75 \pm 3.45$ & $8.70 \pm 1.78$ & $16.65 \pm 6.15$
\end{tabular}

Descriptive statistics (Mean \pm SD) for Forward Digit Span, Backward Digit Span, Selective Attention and London Tower Test among karateka $(n=19)$ and sedentary $(n=20)$ children. 


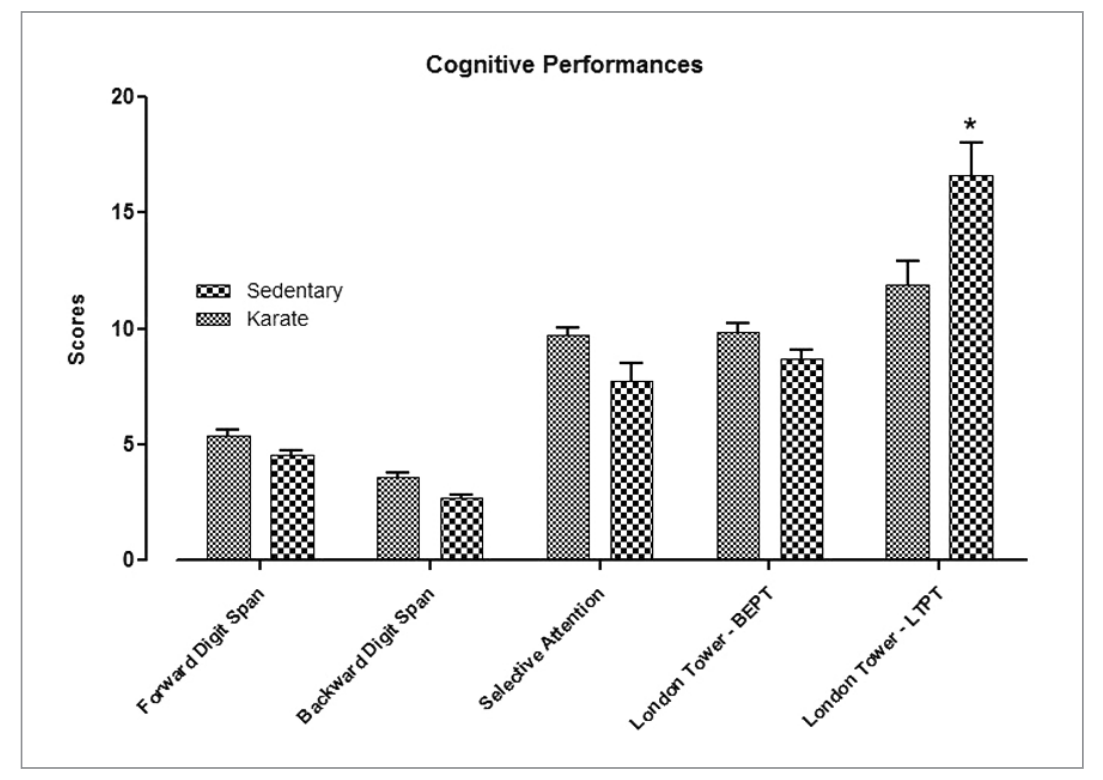

Figure 2. Cognitive abilities of the control group compared to the Karatekas on Selective attention task (TASK); Forward digit span (FDS); Backward digit span (BDS); Better execution of planning task (BEPT); Higher times of planning task (LTPT) $(p<0.05)$. Data are reported as mean and SEM.

\section{Discussion}

Karate is a complete sport activity involving physical as well as psychological components. The training of Karate consists of two components: "Kumitè" is characterized by sparring and combat exercises with the partner and aims at avoiding the disqualification; "Kata" is characterized by forms and sequences of different moves such as a battle against an imaginary antagonist ${ }^{9}$. This discipline allows the osteo-articular and muscular systems work as a whole. At the same time it's considered able to improve self-control and selfawareness ${ }^{16,17}$. Anyway, to our knowledge, few studies showed the connection between motor competences and cognitive profiles in children practicing karate. In our study we aimed at comparing motor and cognitive abilities in children exercising in karate, especially kumitè, three times a week and their sedentary peers. With concern to motor abilities, our study revealed significant differences between children practicing karate and sedentary for all motor tasks. Specifically young karatekas demonstrated to be faster in the run, to have better coordination abilities and to be higher in explosive legs strength than their peers with a sedentary life-style. As expected, our results confirmed the value of regular karate exercise training in increasing speed, coordination and explosive legs strength skills in children. These results are coherent with the motor profile of adult athletes; muscle explosive power, jumping performance and maximal velocity are recognized to have a critical role in the karateka's capacity for high-level performance ${ }^{10,18}$. With concern to cognitive abilities, on the whole, children executing karate appear to have better cognitive functioning. Firstly, they show better performances in visual selective attention task and in verbal working memory. In particular, selective attention abilities sustain the reaction time which plays a core role in karate performance and differentiates athletes and novices. High-level performance is mainly characterized by explosive techniques strictly linked to better choice reaction time ${ }^{18,19}$. The enlargement of concentration and assertiveness is thought to be one of the main results of long-term karate ${ }^{16}$. This effect is confirmed too by clinical studies demonstrating positive effects of karate training to improve sustained attention and reduce impulsivity in children with $\mathrm{ADHD}^{20}$.

The coordination of complex body movements and the adaptation to continually changing exercises sustain top-down cognitive control and decrease automatic behaviours ${ }^{18-20}$. Complementary, our children attending karate scored better on executive functioning than sedentary children. They performed better both in accuracy and in time of execution showing higher performance and lower times on planning tasks. A plausible explanation for this result is the executive function hypothesis supposing that exercise training stimulates a significant rise in gray matter volume and a prolonged increase of myelination and connectivity between age 7 and young adulthood in the pre-frontal and frontal cortex. The refinement of cortical network in this area improves children's components of executive abilities: speed and accuracy of processing, strategy employ, working memory and response inhibition ${ }^{7,20,21}$. Since executive functions are classified as higher-order cognitive abilities, our findings point out the positive influence of regular physical activity, in particular karate, acting via the increasing of the production of neurotrophins, the synaptogenesis and the angiogenesis ${ }^{4}$. Specifically, karate employs exercises in a cognitively-engaging context and entails sophisticated and complex moves which are regulated by the prefrontal neural network.

It requires high cognitive engagement and impacts many of the same processing components as those implied in executive functioning tasks: involving in a 
relevant task, shifting the focus of processing activities, updating of information, monitoring mental representations and inhibiting irrelevant responses. Moreover karate stimulates strategic and goal-directed behaviours when faced to constantly changing situations and movements.

Consistent with previous results, we found in the karate group that the time of visual discrimination correlates positively $(r=0.530)$ with performance on agility test and negatively $(r=-0.583)$ with performance on standing board jump test. It means that karate favors a strong association between motor skills such as coordinative or explosive legs strength skills and underlying processes of perceptual organization such as details recognition in visual images, perceptual grouping, figure-ground segregation and global-local processing. To sum up karate exercise may impact cognitive development through multiple pathways: 1) neuro-physiological by inducing changes in the brain structure; 2) contextual by producing an "enriched environment"; 3) social by creating opportunities of social interaction, cooperation with others, respecting rules, sharing leisure experiences, receiving the encouragement of peers; 4) motivational by increasing self-concept and self-awareness ${ }^{22,23}$. In conclusion, karate practice seems to represent an exercise program holding physical and psychological positive effects during the life span. Diamond and Lee ${ }^{24}$ in their review of interventions able to aid executive function development in children 4 to 12 year old analyze how martial arts produce gains on physical conditioning as well as self-control, inhibitory control, perseverance and math abilities. On the other hand adapted karate trainings are proved to slow the process of aging by increasing physiological, psychological and social dimensions during the old age $^{12}$. Anyway it is important to acknowledge that exercise interventions are complex and need to respect at least two principals: specificity and over-loading. A feature of the training is the specificity, which improves substantially the only function that is trained (cardiorespiratory fitness, muscular strength, muscular endurance or flexibility), usually at the expense of the function which, from physiological point of view, has the opposite characteristics. So for if you want to improve muscle strength, which implies an increase in muscle mass, this comes at the expense of agility. Another general feature of the training is the progressive overload binding various systems to an higher level than normal. The choice of the load must be proportionate to the physiological condition of the subject and should be targeted at a realistic goal. In this perspective research employing karate exercise training show global benefits resulting in biological changes in both muscles and organs that, in turn, modify and regulate the structure and the functioning of the brain ${ }^{19}$. The findings of this study about motor and cognitive abilities in physically fit and sedentary children highlight the need for this issue to be addressed in future research. In particular they suggest some interesting implications on the sport and educational field. It might be worth looking further into the possibility to plan and realize screenings aimed at identifying young karatekas characterized by good abilities of planning, sustained and divided attention, visual and verbal working memory, inhibition of previous responses, all those abilities relevant for an highlevel athlete in the future.

Coherently, from a psycho-educational perspective, it's crucial to organise settings aimed at encouraging the sport participation. The relation between exercise and children's health has not, until relatively recently, been systematically evaluated. It's important for researchers and practitioners to understand what exercise interventions are needed to influence the whole development not only in the motor and physical domain but even in the cognitive area. Direct implications would apply to public health concerning the limitation of overweight and obesity associated to the increasing hypokinetic life-style during childhood ${ }^{25}$. It is crucial to acknowledge that future research is needed building on the shortcoming of this study. The main goal is to provide a richer and more complete understanding of how different sport disciplines would affect child development.

Again it would be interesting to deepen motor and cognitive profiles of children exercising different components of karate such as kumitè and kata characterized by different features (open or closed skill activities), different goals and different exercise training programs.

\section{Acknowledgments}

We are grateful to all participants to the study and their families.

\section{Conflict of interest}

None.

\section{Funding}

No funding was received for this article.

\section{Authors Contribution}

All authors contributed equally to this manuscript.

\section{References}

1. Chomitz VR, Slining MM, McGowan RJ, Mitchell SE, Dawson GF, Hacker KA. Is There a Relationship Between Physical Fitness and Academic Achievement? Positive Results From Public School Children in the Northeastern United States. J Sch Health. 2009;79(1):30-37.

2. Nakata H, Yoshie M, Miura A, Kudo K. Characteristics of the athletes' brain: Evidence from neurophysiology and neuroimaging. Brain Res Rev. 2010;62:197-211. 
3. Babiloni $\mathrm{C}$, Marzano N, Infarinato F, lacoboni M, Rizza G, Aschieri P, Cibelli G, Del Percio C. "Neural efficiency" of experts' brain during judgment of actions: A high-resolution EEG study in elite and amateur karate athletes. Behav Brain Res. 2010; 207(2): 466-475.

4. Best JR. Effects of Physical Activity on Children's Executive Function: Contributions of Experimental Research on Aerobic Exercise. Dev Rev. 2010;30(4):331-551.

5. Tomporowski PD, Davis CL, Miller PH, Naglieri JA. Exercise and Children's Intelligence, Cognition, and Academic Achievement. Educ Psychol Rev. 2008;20(2):111-131.

6. Alesi M, Battaglia G, Roccella M, Testa D, Palma A, Pepi A. The improvement of gross-motor and cognitive abilities by an Exercised Training Program; three case reports". Neuropsychiatr Dis Treat (in press).

7. Best JR, Miller PH. A Developmental Perspective on Executive Function. Child Dev. 2010;81(6):1641-1660.

8. Burke DT, Al-Adawi S, Lee YT, Audette G. Martial arts as sport and therapy. J Sports Med Phys Fitness. 2007;47:96-102.

9. Jansen P, Dahmen-Zimmer K. Effects of cognitive, motor and karate training on cognitive functioning and emotional well-being of elderly people. Front Psychol. 2012;3:40-41.

10. Chaabène $H$, Hachana $Y$, Franchini $E$, Mkaouer $B$, Chamari $K$ Physical and Physiological Profile of Elite Karate Athletes. Sports Med. 2012;42(10):829-843.

11. Etnier JL. A meta-regression to examine the relationship between aerobic fitness and cognitive performance. Brain Res Rev. 52;(1): 119-130.

12. Chateau-Degat M, Ludivine G, Lopez A. Effect of adapted karate training on quality of life and body balance in 50-yearold men. Open Access J Sports Med. 2010;1:143-150.

13. Mori S, Ohtani $Y$, Imanaka K. Reaction time and anticipatory skills of Karate athletes. Hum Mov Sci. 2002;21(2):213230.

14. Padulo J, Oliva F, Frizziero A, Maffulli N. Muscles, Ligaments and Tendons Journal. Basic principles and recommendations in clinical and field science research. MLTJ. 2013:4:250-252.
15. Bisiacchi PS, Cendron M, Gigliotta M, Tressoldi PE, Vio C Batteria di Valutazione Neuropsicologica per l'Età Evolutiva. 2005;Trento; Erickson.

16. Conant KD, Morgan AK, Muzykewicz D, Clark DC, Thiele EA A karate program for improving self-concept and quality of life in childhood epilepsy: Results of a pilot study. Epilepsy Behav. 2008:12:61-65.

17. Vando S, Filingeri D, Maurino L, Chaabène H, Bianco A, Salernitano G, Foti C, Padulo J. Postural adaptation in preadolescent karate athletes due to a one week karate training cAMP. J Hum Kinet. 2013;38:45-52.

18. Azaiez F, Chalghaf N, Cherif E, Achour K, Souissi C. Evaluation of the Mental Skills of the High Level Athletes: Example of the Athletes of Martial Arts. IOSR-JHSS. 2013;10 (4):58-65.

19. Diamond $A$. The interplay of biology and the environment broadly defined. Dev Psychol. 2009; 45:1-8.

20. Leggett C, Hotham E. Treatment experiences of children and adolescents with attention-deficit/hyperactivity disorder. J Paediatr Child Health. 2011;47(8):512-517.

21. Barenberg J, Berse T, Dutke S. Executive functions in learning processes: Do they benefit from physical activity? Educational Research Review. 2011;6:208-222.

22. Alesi M, Pepi A. Self-esteem and Self-perception profile; a comparison between children attending sport and sedentary children. EJSS Journal. 2013;1(2):102-112.

23. Battaglia G, Alesi M, Inguglia M, Roccella M, Caramazza G, Bellafiore M, Palma A. Soccer practice as an add-on treatment in the management of individuals with a diagnosis of schizophrenia. Neuropsychiatr Dis Treat. 2013;9:595-603.

24. Diamond A, Lee K. Interventions shown to aid executive function development in children 4 to 12 year old. Science. 2012;333:959-964

25. Burkhalter TM, Hillman $\mathrm{CH}$. A Narrative Review of Physical Activity, Nutrition, and Obesity to Cognition and Scholastic Performance across the Human Lifespan. Adv Nutr. 2011; 2:2015-2020. 